Gegner dominierten Länderkammer haben allerdings dazu beigetragen, in der Föderalismusreform einen anderen Weg zu gehen: die Stärkung der eigenständigen Gesetzgebungszuständigkeiten von Bund und Ländern. Ob diese - partielle - Entflechtung der Gesetzgebungszuständigkeiten in der politischen Praxis spürbar wird und ob sie die Rolle des Bundesrats schwächt, bleibt allerdings abzuwarten.

Unverändert verteidigt Reuter das „freie Mandat“ der Landesregierungen gegenüber der Einflussnahme der Landesparlamente. Diese können den Regierungen keine verbindlichen Weisungen für die Stimmabgabe im Bundesrat geben; das ist unumstritten. Entgegen der Ansicht von Reuter dürften unverbindliche - oder besser gesagt, „nur“ politisch verbindliche - „Empfehlungen“ oder „Orientierungshilfen“ dagegen unproblematisch sein, denn die parlamentarische Kontrolle einer Landesregierung erstreckt sich selbstverständlich auch auf deren Verhalten im Bundesrat. Deshalb kann das Landesparlament genauso wie zum Beispiel zum Gesetzesvollzug, zu politischen Programmen oder zu administrativen Einzelfällen Wünsche, Anregungen und Forderungen an die Landesregierung richten. Und genauso selbstverständlich entscheidet die Regierung nach eigenem politischen Ermessen - und auf eigenes politisches Risiko -, inwieweit sie ihnen folgt. Zu praktischen Problemen führt das in der Regel nicht: Zum einen stimmt sich die Landesregierung mit der sie tragenden Mehrheit im Parlament ohnehin über ihr Verhalten im Bundesrat ab, wenn es um politisch bedeutsame Fragen geht. Zum anderen fehlt den Landtagen die Arbeitskapazität, um die Bundesratspolitik umfassend zu kontrollieren und zu begleiten.

Die aufgeführten Punkte zeigen, dass Reuter pointiert Positionen bezieht zugunsten des Bundesrats. Sie regen an zur Diskussion, und das ist für ein wissenschaftliches Werk ein Kompliment. Im Übrigen bleibt Reuter der zuverlässige und gründliche Kommentator nicht nur der einschlägigen verfassungsrechtlichen Grundlagen, sondern auch der Geschäftsordnung des Bundesrats.

$\mathrm{Zu}$ begrüßen ist das ausführliche Sachverzeichnis, es erleichtert das Nachschlagen beträchtlich. Was den Rezensenten allerdings stört, nicht nur in Reuters Handbuch, ist die Unsitte, mit Fettdruck einzelne Wörter oder Passagen hervorzuheben. Der gut aufgebaute und gegliederte Text gibt dem Leser ausreichend Orientierung.

Im Geleitwort hat der damalige Bundesratspräsident Peter Harry Carstensen das Handbuch als „unverzichtbaren Wegweiser“ mit hohem Nutzen für die Praxis und Wissenschaft bezeichnet. Dem kann sich der Rezensent nur anschließen.

Florian Edinger

\title{
Politische Strategie: Grundlegung für Forschung und Praxis
}

Raschke, Joachim und Ralf Tils: Politische Strategie. Eine Grundlegung, VS Verlag für Sozialwissenschaften, Wiesbaden 2007, 585 Seiten, $€ 39,90$.

„Wenn man erst einmal als Machtmensch angesehen wird, der alles kühl plant, gerinnt der Zufall zur Strategie. Doch häufig ist es weit weniger Strategie, als man es vermutet", so wurde Gerhard Schröder in der 1996 publizierten Biographie zitiert ${ }^{1}$. Möglichkeiten und 
Grenzen von Strategien in der Politik - dies illustriert das Zitat des späteren Bundeskanzlers - sind schwer zu erfassen. Joachim Raschke und Ralf Tils haben sich in ihrem Buch mithin eines hoch komplexen, in der deutschsprachigen Politikwissenschaft zudem bislang eher am Rande behandelten Gegenstands angenommen. Beide Autoren knüpfen an früher vorgelegte Arbeiten an, verbinden mit dem nun erschienenen, über 500 Seiten umfassenden Werk jedoch den Anspruch einer grundlegenden Konzeptualisierung politischer Strategien wie auch eines Beitrags zur Kritik und Optimierung strategischer Praxis.

Entgegen aller Skepsis gegenüber Strategien in der Politik konstatieren Raschke und Tils einen „objektiv wachsende(n) Strategiebedarf“ (S. 11). Ihren Untersuchungsgegenstand verorten die Autoren in der empirischen wie praktischen Politikwissenschaft. Sie stützen sich daher nicht nur auf Literatur - auch aus benachbarten Disziplinen -, sondern außerdem auf eine Reihe von Interviews und Hintergrundgesprächen mit Praktikern und Experten. Der Fokus der Studie liegt auf Kollektivakteuren in der Innenpolitik westeuropäischer PartyGovernment-Systeme, konkret auf Parteien, Parlamentsfraktionen und Regierungen.

Die Autoren gehen in drei Schritten vor: Zunächst widmen sie sich den Grundlagen der Strategieforschung. Ausgehend von einem empirisch gestützten Grundmodell entwerfen sie ein Gesamtkonzept der Strategieanalyse. Anschließend setzen sie sich mit Aspekten des Strategy-Makings “ auseinander, das von der Strategiefähigkeit über die Strategiebildung bis zur strategischen Steuerung reicht. Danach werden kompakte Fallstudien zum Strategy-Making der SPD präsentiert, die bis in das Jahr 1958 zurückreichen und Phasen der Kohärenz oder Fragmentierung erkennen lassen. Das Fazit plädiert für einen neuen Ansatz der politischen Strategieanalyse und unterbreitet Optimierungsvorschläge für die politische Praxis.

Ein Charakteristikum des Buches ist die elaborierte, in wesentlichen Teilen neu eingeführte und hoch ausdifferenzierte Begrifflichkeit. Damit leistet es einen wertvollen Beitrag zur Überwindung der inflationären, aber oftmals unscharfen Verwendung des Strategiebegriffs. Die im Vorwort schon angekündigte „Anstrengung des Begriffs“ stellt tatsächlich eine gewisse Herausforderung für den Leser dar. Äußerst hilfreich sind daher die tabellarischen und grafischen Darstellungen, welche die begrifflich-konzeptionellen Zugänge fortlaufend veranschaulichen.

Raschke und Tils, dies ist hervorzuheben, vertreten einen intentionalen Strategiebegriff, wenden sich also bewusst gegen Vorbehalte der Zufälligkeit oder Undurchführbarkeit. Strategien, so die Kerndefinition, sind „erfolgsorientierte Konstrukte, die auf situationsübergreifenden Ziel-Mittel-Umwelt-Kalkulationen beruhen“ (S. 127). Die Begriffsklärung unterscheidet zwischen strategischem, operativem und taktischem Handeln, betont jedoch ausdrücklich, dass Strategien auch kurzfristig, zum Beispiel für einen Parteitag, angelegt sein können. Das Strategiekonzept beinhaltet außerdem ein Orientierungsschema, das in einem generalisierenden Zugriff zentrale Parameter und Referenzpunkte strategischer Politik systematisiert (vgl. die Abbildung S. 163). Erwähnt sei hier lediglich die Grundannahme, dass der Referenzrahmen der Akteure maßgeblich durch das Spannungsverhältnis zwischen Problem- und Konkurrenzpolitik unter Bedingungen der Öffentlichkeit bestimmt wird.

Das Drei-Säulen-Modell des Strategy-Makings - Strategiefähigkeit, -bildung und strategische Steuerung - kennzeichnet Strategiefähigkeit als Schlüsselfaktor. Grundlegend hierfür sind nach Raschke und Tils die Elemente Führung, Richtung und Strategiekompetenz. Von besonderer Bedeutung erscheint hier der Aufbau eines leistungsfähigen „strategischen Zentrums“. Dabei handelt es sich um ein informelles, nur wenige Personen umfassendes Netzwerk, das über Strategien entscheidet und steuert. Zu berücksichtigen ist auch der Unter- 
stützungskomplex des strategischen Zentrums, vor allem das Zusammenspiel mit der operativen Leitung und dem strategischen Apparat sowie das weitere Beratungsumfeld. Grundsätzlich machen die Autoren hier Trends der Zentrierung, Entideologisierung und Professionalisierung aus. Dabei wird nicht übersehen, dass die institutionellen Ausprägungen des jeweiligen politischen Systems, insbesondere die Führungskonstellationen der Parteiendemokratie, die Chancen zur Etablierung eines funktionstüchtigen „strategischen Zentrums“ wesentlich bedingen. Hinzu kommen kontingente Faktoren: „Führungsfragen werden meist nicht strategisch entschieden.“ (S. 293) Zwar richtet sich das Strategiekonzept der Autoren auf Kollektivakteure, doch verdeutlichen die empirischen Fallstudien verwiesen sei hier nur auf den situativen Politikstil von Bundeskanzler Schröder - klar die Relevanz der handelnden Personen und ihres individuellen Führungsstils.

In der Strategiebildung, der zweiten Säule des Strategy-Makings, geht es vorrangig um die Qualität strategischer Konzepte. Hier erkennen die Autoren ein unausgeschöpftes Optimierungspotenzial in der Methodik und den Verfahrensweisen. Die Säule der strategischen Steuerung betrifft schließlich den Prozess der Umsetzung einer konzipierten oder sich (graduell) herausbildenden Strategie. Diese umfasst Organisations-, Problempolitik-, Konkurrenzpolitik- und Kommunikationssteuerung. Hervorzuheben ist, dass die Autoren keineswegs einem „Planungsirrtum“ unterliegen, sondern die permanente Anpassung von Strategien im dynamischen Politikprozess für erforderlich halten.

Der von Raschke und Tils entfaltete Ansatz politischer Strategieanalyse erweist sich in doppelter Hinsicht als anschlussfähig. Der Praxis liefert er zwar kein Rezeptwissen, kann aber einen Beitrag dazu leisten, das eigene, täglich praktizierte Handeln zu überprüfen und zu strukturieren. Auch dürfte das Buch auf Jahre hinaus Bestand haben als Grundlegung der politikwissenschaftlichen Strategieforschung. Es verweist auf Forschungsdesiderata und liefert wichtige Impulse, theoretisch wie empirisch weiter an Strategiefragen zu arbeiten.

Manuela Glaab

\section{Parteien in Deutschland: kompetentes und informatives Handbuch}

\section{Decker, Frank und Viola Neu (Hrsg.): Handbuch der deutschen Parteien, VS Verlag für Sozial- wissenschaften, Wiesbaden 2007, 440 Seiten, $€ 29,90$.}

Das deutsche Parteiensystem hat sich in den letzten Jahren im nationalen, regionalen und kommunalen Raum ausdifferenziert. Immer mehr Parteien gewinnen auf den verschiedenen Ebenen des politischen Systems an parlamentarischer Präsenz, wodurch sich die Handlungsoptionen der beteiligten Akteure und ihr Verhältnis untereinander verändern. Wie nicht zuletzt der Ausgang der Bundestagswahl 2005 verdeutlicht hat, erschwert die Fragmentierung des Parteiensystems die herkömmlichen (kleinen) Zweierkoalitionen und in der Folge die Regierungsbildung. Diese Entwicklung lenkt das Interesse der Wissenschaft zunehmend auf die kleinen Parteien. So hat der Arbeitskreis Parteienforschung der Deutschen Vereinigung für Politische Wissenschaft bereits im Jahre 2005 seine Jahrestagung diesem Thema gewidmet. Auch für das von Frank Decker und Viola Neu herausgegebene Parteienhandbuch ist die Auffächerung des Parteiensystems und der Bedeutungs- und Einflussgewinn der kleinen Parteien Ausgangspunkt der Betrachtungen. Die Herausgeber wol- 